\title{
Virtual reality applications in manufacturing system
}

\begin{abstract}
Virtual reality (VR) is a high-end human computer interface that strives to immerse the designers and users completely in a virtual interactive environment for a simulation of real world. In order to meet the requirements of market competition, VR technologies can not only reduce effectively the time and cost, but also optimize complex products in the design process. Virtual reality is a rapidly developing computer interface that strives to immerse the user completely within an experimental simulation, thereby enhancing the overall impact and providing a much more intuitive link between the computer and the human participants. Virtual reality has been applied successfully to hundreds of scenarios in diverse areas including prototyping, manufacturing, scientific visualisation, engineering, and education. This paper discusses the applications of virtual reality in the manufacturing industry.
\end{abstract}

Keyword: Virtual reality; Virtual manufacturing; Simulation 